- Corticosteroids are used widely in maxillofacial surgery.

- Psychosis may result from brief corticosteroid regimens.

- Consultant surgeons may exercise holding power to keep 'at risk' patients in hospital.

- Input from a consultant psychiatrist is vital to ensure a satisfactory outcome.

\title{
Steroid-induced psychosis complicating orthognathic surgery: A case report
}

\author{
P. S. Fleming ${ }^{1}$ and T. R. Flood ${ }^{2}$
}

Psychosis is an uncommon but well-recognised complication of short-term corticosteroid administration. We report a case of steroid-induced psychosis subsequent to a bimaxillary osteotomy.

\begin{abstract}
INTRODUCTION
Corticosteroids were first available for commercial use in $1950 .{ }^{1}$ A link between steroids and psychiatric sequelae emerged later that year. ${ }^{2}$ Peri-operative steroids help to limit post-operative oedema, prevent airway compromise, suppress inflammation and discomfort, and may reduce postanaesthetic nausea. ${ }^{3}$ Shortterm corticosteroids form part of the mainstay of pharmacological management of patients undergoing maxillofacial surgery. However, even short-term use of steroids is not without potential complications. ${ }^{4}$ We present a case of steroidinduced psychosis arising six days subsequent to a bimaxillary osteotomy.
\end{abstract}

\section{CASE REPORT}

A 16-year-old female with a severe Class 2 skeletal discrepancy and marked vertical maxillary excess was admitted for a bimaxillary osteotomy. She had no relevant medical or psychiatric history. There was an unsubstantiated family history of a

1"Senior House Officer, Ballina Road, Enniscrone, Co. Sligo, Ireland; ${ }^{2}$ Consultant, Odstock Centre for Burns, Plastic \&t Maxillofacial Surgery, Salisbury District Hospital, Odstock Rd, Salisbury SP2 8BJ.

${ }^{*}$ Correspondence to: Dr Padhraig Fleming

Email:padhraigfleming@hotmail.com

\section{Refereed Paper}

Accepted 14 June 2005

doi: 10.1038/sj.bdj.4812929

๑ British Dental Journal 2005; 199: 647-648 bleeding tendency. Routine pre-operative haematological investigations failed to show any underlying abnormality. A bilateral sagittal split osteotomy with a subapical Kole procedure and a Le Fort 1 osteotomy were performed to correct the underlying skeletal discrepancy.

The patient was administered our routine steroid regimen of $250 \mathrm{mg}$ methylprednisolone intravenously at induction followed by three further doses of $250 \mathrm{mg}$ methylprednisolone at six-hourly intervals.

Post-operative recovery was hampered by profuse and prolonged bleeding and facial swelling, necessitating admission to the intensive care unit. Estimated blood loss of 2.5-3 litres occurred peri-operatively. The patient was transfused with four units of blood, as well as crystalloid and colloid. She remained intubated and deeply sedated with midazolam and propofol in the intensive care unit for three nights.

Following extubation she returned to the ward where her recovery was initially uneventful. On the sixth post-operative day she began to elicit signs of psychiatric disturbance. The consultant surgeon in charge exercised his 'holding power' in accordance with the Mental Health Act 1983 to detain the patient in the interests of her own health and safety. A psychiatrist examined the patient to confirm the 'Three Day Order' and to oversee further care.

The patient displayed an array of psychiatric manifestations including delusion, hallucinations and disorientation. Delusions of bodily change were reported including a conviction that the surgery required repeating, that sperm were multiplying on her face and a belief that she had become pregnant. She developed marked paranoia believing that the heating system was transmitting her thoughts. Auditory hallucinations were also reported as she was convinced she could hear doctors talking about her. She also believed she had ultrasonic hearing and psychic powers. An organic cause for this acute psychiatric episode was first suspected. Biochemical investigations revealed no abnormality and it became clear that the condition was steroid-induced. Initial treatment with chlorpromazine, a Group 1 phenothiazine, $25 \mathrm{mg}$ three times daily and $50 \mathrm{mg}$ as required in acute phases to a maximum overall daily dose of $300 \mathrm{mg}$ was commenced.

The medication failed to produce immediate improvement. She continued to have symptoms of visual hallucinations, disorientation, memory impairment and a variable mental state. She described vivid dreams of human brain hijacking and felt compelled to commit suicide. Later she developed oculogyric crisis; an acute dystonic reaction to the chlorpromazine. She was prescribed procyclidine hydrochloride to treat these extra-pyramidal side effects. During her lucid phases she appeared to be developing some insight into her condition. 
On the tenth post-operative day she relapsed, discharging herself from the ward and almost becoming involved in a road traffic accident. Thereafter the patient's parents volunteered to mind her continually at her bedside. Her chlorpromazine prescription was altered. Over the next two days she finally recovered, developing an insight into her condition, becoming well orientated and thinking more clearly. She was discharged on the fourteenth post-operative day. The chlorpromazine was continued for a further two weeks. Further psychiatric review indicated a complete emotional recovery.

\section{DISCUSSION}

Corticosteroids play an integral role in peri-operative management of maxillofacial patients. ${ }^{5}$ Their main role involves limitation of swelling at the surgical site. The primary factor in development of postoperative oedema involves an increase in water content in damaged tissue. ${ }^{6}$ Prolonged vascular leakage of fluid, which is thought to culminate in oedema, may be prevented with steroid intervention. This is achieved by stabilisation of cell membranes and modification of the inflammatory response. Degranulation of leukocytes is suppressed, permeability of endothelial cells is reduced, and adherence of leukocytes to endothelial cells is decreased.

Short courses of steroids are quite commonly deployed in maxillofacial practice for removal of wisdom teeth and for facial osteotomies. ${ }^{7}$ Convincing evidence of their efficacy in reduction of postoperative swelling and protection of masticatory function following wisdom tooth removal exists. The data supporting steroid use in peri-operative management of osteotomies are difficult to prove. ${ }^{8}$ However, the literature does suggest that systemic methylpredisolone or dexamethasone does result in less postoperative facial oedema. ${ }^{9}$ Some authors also advocate the use of steroids in parotidectomy and thyroidectomy pointing to a putative neuroprotective role provided by the limitation in swelling. ${ }^{10}$

Different corticosteroid regimens for peri-operative management of orthognathic patients are available. Surgical trauma may result in endogenous secretion of up to $300 \mathrm{mg}$ hydrocortisone daily. Therefore, for maximum efficacy exogenous steroids exceeding $300 \mathrm{mg}$ hydrocortisone must be administered. As yet no particular corticosteroid regime has gained widespread acceptance. ${ }^{11}$ Dexamethasone is often used as it has minimal mineralocorticoid activity, profound anti- inflammatory effects and a long duration of action. Methylprednisolone is an intermediate-acting agent with a half-life of 18 to 36 hours. Prior to this case we had used the methylprednisolone regimen without any significant side effects for many years.

Prolonged use of corticosteroids may exaggerate some of their normal physiological actions producing mineralocorticoid and glucocorticoid side effects. Mineralocorticoid effects include hypertension, water retention, hypernatraemia and hypokalaemia. Detrimental glucocorticoid changes include diabetes, osteoporosis, and proximal myopathy. The link between prolonged steroid treatment and a plethora of psychiatric disturbances including depression, manic symptoms, euphoria, psychosis and delirium is well-documented. ${ }^{12}$

Short regimens are not without side effects. Steroid-induced acne secondary to short-term steroid administration following maxillofacial procedures has been reported. ${ }^{13}$ Adrenal suppression is of little significance in short-term therapy, however. ${ }^{14}$ The connection between brief regimens and mental disturbances has also been documented. A case of steroid psychosis culminating in attempted murder of a spouse following a short steroid course has been reported. The patient was acquitted because the judge ruled that a steroidinduced altered mental state pre-empted the attack. ${ }^{15}$ Steroid-induced psychiatric disturbance has also been reported following a hemi-maxillectomy in a patient who already took tricyclic antidepressants and benzodiazepines. ${ }^{16}$ Our patient had no previous psychiatric history.

Corticosteroid-induced psychosis is related to neurocytotoxic effects of raised cortisol levels. While there is substantial evidence to indicate that the hippocampus is particularly sensitive to elevation of glucocorticoids, the effects on other areas of the brain are less clear. A recent study indicated that the frontal lobe is adversely affected by cortisol, which may illustrate a similar pattern of neural degeneration to that which occurs in the hippocampus. ${ }^{17}$ Three independent studies have also raised the possibility of specific corticosteroidrelated cognitive impairments. ${ }^{18}$

Steroid-induced psychosis should be considered in patients with no psychiatric history administered high dose, potent steroids with long half-lives. Our case very nearly resulted in tragic loss of life from attempted deliberate self harm. Liaison with the patient's family helped to protect the patient. Because of its potential severity the need for prompt recognition and treatment cannot be overstated. Involvement of the psychiatric team is essential in ensuring accurate diagnosis and appropriate management.

Brief courses of steroids have undisputed benefits in maxillofacial surgery. The evidence supporting peri-operative use in orthognathic surgery is less compelling. Double-blind prospective research is needed to highlight benefits of peri-operative steroids in such cases. Recent advances in accurate measurement of facial swelling may facilitate this. ${ }^{18}$

1. Rome H P, Braceland FJ. The psychological response to ACTH cortisone, hydrocortisone, and related steroid substances. Am J Psychiatry 1952; 108: 641-651.

2. Rome H P, Braceland FJ. Use of cortisone, and ACTH in certain diseases: psychiatric aspects. Proc Staff Meet Mayo Clinic 1950; 25: 495-497.

3. Sisk $A$, Bonnington $F J$. Evaluation of methylprednisolone and flurbiprofen for inhibition of the postoperative inflammatory response. Oral Surg 1985; 60: 137-145.

4. Galen D M, Beck M, Buchbinder D. Steroid psychosis after orthognathic surgery: a case report. J Oral Maxillofac Surg 1997; 55: 294-297.

5. Flood T R, McManners J, El Attar A, Moos K F. Randomized prospective study of the influence of steroids on postoperative eye-opening after exploration of the orbital floor. J Oral Maxillofac Surg 1999; 37: 316-319.

6. Bertolami C N, Cohen $\mathrm{CH}$, Chrzanowsk R R. Quantitation of post-surgical oedema. J Oral Maxillofac Surg 1982; 40: 473-476.

7. Gersama L, Baker K. Use of corticosteroids in oral surgery. J Oral Maxillofac Surg 1992; 50: 270-277.

8. Munro IR, Boyd J B, Wainright D J. Effects of steroids in maxillofacial surgery. Ann Plast Surg 1986; 17: 440-444.

9. Schaberg S J, Stuller C B, Edwards S M. Effects of methylprednisolone on swelling after orthognathic surgery. J Oral Maxillofac Surg 1984; 42: 356-361.

10. Ferris R L, Eisele D W. Steroid psychosis after head and neck surgery: Case report and review of the literature. Otolaryngol Head Neck Surg 2003; 129: 591-592.

11. Guernsey L H, DeChamplain R W. Sequelae and complications of the intraoral sagittal split osteotomy in the mandibular rami. Oral Surg 1971; 32: 176-192.

12. Sirios F. Steroid psychosis: a review. General Hospital Psychiatry 2003; 25: 27-33.

13. Precious D, Hoffman C D, Miller R. Steroid acne after orthognathic surgery. Oral Med Oral Surg Oral Pathol 1992; 74: 279-281.

14. Butler R C D, Vorono A A, Finstuen K. Dosage effects of pulsed steroid therapy on serum cortisol levels in oral and maxillofacial surgery patients. J Oral Maxillofac Surg 1993; 51: 750-753.

15. D'Orban P T. Steroid-induced psychosis. Lancet 1989; 2: 694.

16. Silva R G, Tolstunov L. Steroid-induced psychosis: Report of case. J Oral Maxillofac Surg 1995; 53: 183 186.

17. Young A H, Sahakian B J, Robbins T W, Cowen P J. The effects of chronic administration of hydrocortisone on cognitive function in normal male volunteers. Psychopharmacology (Berl) 1999;145: 260-266.

18. Wolkowitz $\mathrm{OM}$, Reus VI, Weingartner $\mathrm{H}$ et al. Cognitive effects of corticosteroids. Am J Psychiatry 1990; 147: 1297-1303.

19. Harrison J A, Nixon M A, Fright W R, Snape L. Use of hand-held laser scanning in the assessment of facial swelling: a preliminary study. Br J Oral Maxillofac Surg 2004; 42: 8-17. 